\title{
Status Report on Factoring (At the Sandia National Laboratorles)*
}

James A. Davis, Diane B. Holdridge and Gustavus J. Simmons

Sandia National Laboratories

Albuquerque, New Mextco 87185

\section{Introduction}

It is well known that the cryptosecurity of the RSA (R1vest-ShamirAdleman) two key cryptoalgorithm [1] is no better than the composite modulus is difficult to Eactor. Except for one special case, the converse statement is still an open and extremely important question. It is not so well known, perhaps, that there are several other crypto-like schemes whose performance is also bounded by the difficulty of factoring large numbers: the digital signature schemes of Ong-Schnorr [2], of Ong-Schnorr-Shamir [3] and of Schnorr [4], the oblivious transfer channel of Rabin [5] and the subliminal channel of Simmons [6] to name only a few. The point is that the difficulty of factoring large integers has become a vital parameter in estimating the security achievable in many secure data schemes -- and conversely factoring techniques are potentially a tool for the cryptanalyst if the cryptographer misjudges the difficulty of factoring a composite number on which he bases a system.

The Sandia National Laboratorles have already flelded several secure data systems that are dependent on the difficulty of factoring for their security $[7,8,9]$ and at least as many other applications are approaching

* This work performed at Sandia National Laboratorles supported by the U. S. Department of Energy under contract No. DE-AC04-76DP00789. 
reallzation. As a result, a concerted research effort was intitated in 1982 In the Mathematics Department at Sandia to define as sharply as possible the bounds on the computational feasibility of factoring large numbers, using the most powerful computers avallable -- as efficiently as possible -- with the factoring algorithms being carefully matched to the architecture of the machine on which the algorithm was to be run [10]. Our primary objective In this paper will be to present an overview of the advances in factoring resulting from this research. Later, we shall discuss in detail the mathematical and coding advances themselves. Suffice it to say that a roughly three-order of magnitude improvement in factoring -- as measured by the time required to factor a particular size number -- has been achieved by the Sandia researchers over what was possible (and well benchmarked) a few years ago. This is a comblned effect due in part to a new generation of computers with much increased computing power and especially due to the unique architecture of the Cray family of machines, in part due to substantial advances in factoring algorithms and finally -- and equally significant - In part attributable to the efficlency with which the algorithms have been coded for the specific computers. Our secondary objective w11 be to separate out the contributions of these three factors (to factoring progress) in order to both understand how the improvements of the past three years were achieved as well as to project what the state of the art in factoring is likely to be 5 to 10 years from now.

\section{An Overview}

The easiest question to ask concerning lnteger factoring and the hardest to answer, is; "How large a number is 1t computationally feasible to factor using a general purpose factoring routine?" Figure 1 gives one 
number to

b factored

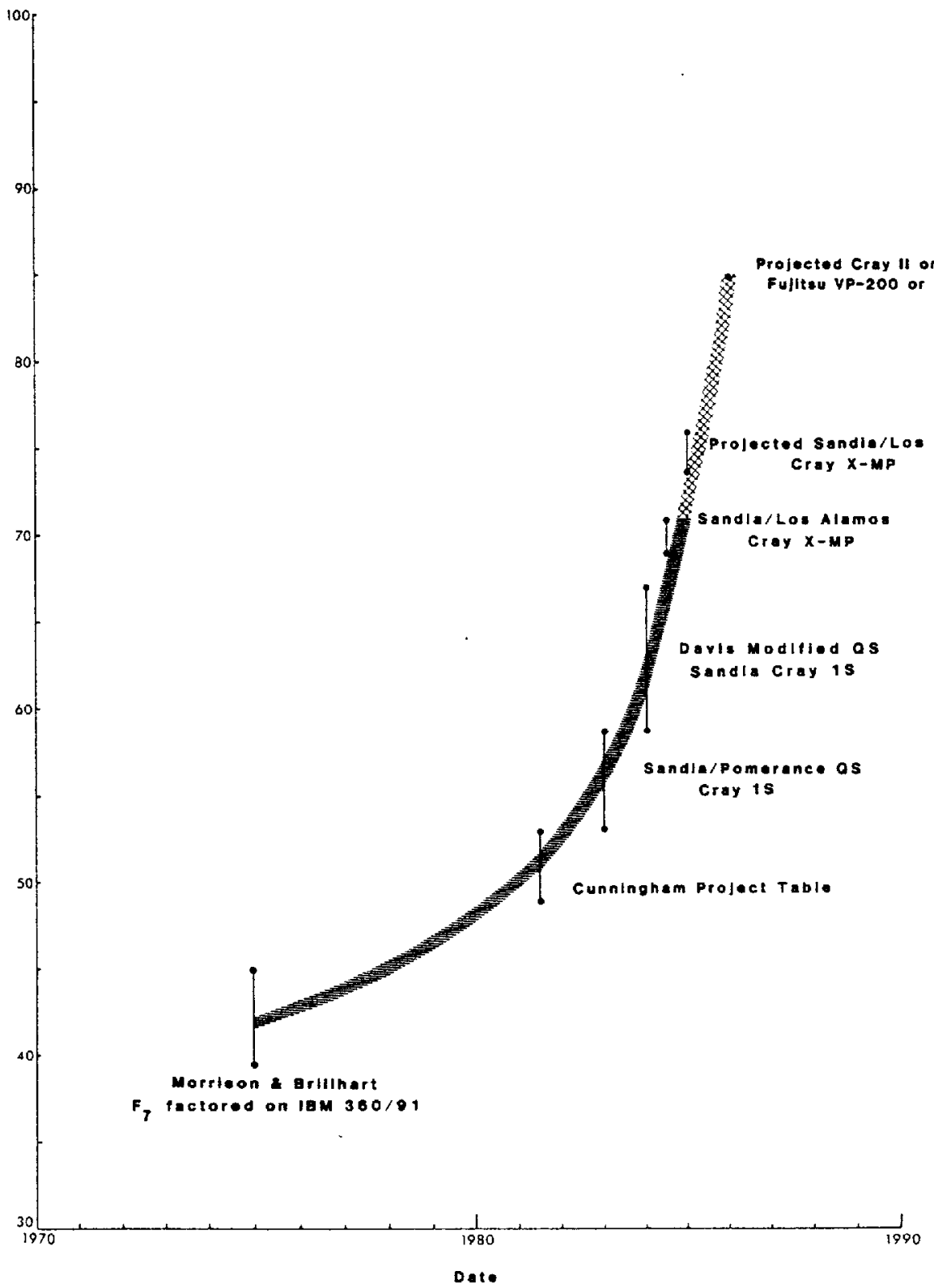

Figure 1. 
answer showing the record size numbers that were factored as an approximate function of the year in which the factorization occurred -- over a perfod of roughly a decade. The data in Figure 1 were selected as being the most Indicative of the state of the art in factoring at the time, elther because the factorization was general1y acknowledged as a major achievement or advance or, as in the case of the Cunningham Table Project [11], because of the thoroughness with which the benchmark was defined. The reader should be aware, however, that these data points are virtually impossible to cross-compare. Within the Sandia data alone the times required to factor the reported numbers range from 7.2 minutes to 32.3 hours, involve three generations of continuously changing computer codes and were run on elther a Cray is or a Cray X-MP computer. The other data have even more variability. The algorithms were run on widely different machines -- some In a dedicated computing environment and others in a time sharing mode. In some instances, the total time required was reported, in others only that time required for the part of the algorithm that was of primary concern to the research. At this late date, there is no way that these results can be "normalized" to make them directly comparable. Instead, we exhibit Figure 1 as the best indicator we have of the progress in factoring during the past decade -- with a strong caution to the reader to not try to read more into (or from) the flgure than this.

The first data point is the landmark factorization of $F_{7}$, a 39-digit number, by Morrison and Brillhart [12] using their continued fraction algorithm. This algorithm was to become the progenitor of a series of steadily improving continued fraction algorithms that dominated the factoring scene unt11 Pomerance's quadratic sleve [13] was first Implemented at Sandfa in 1982 [10]. Using an IBM 360/91 over a period of several weeks, 
Morrison and Brillhart found the necessary number of completely factored quadratic residues in a total CPU time of only 90 minutes. They don't report the time required to carry out the Gaussian elimination (In 2700 variables - primes) but it must have been large. Based on recent discussions with John Brillhart, it appears that their technique and machine could have factored numbers in the mid-forty digtt range in times comparable to the average of the times required for the two dozen or so factorizations that make up the Sandia data points. The error bar on the Morrison and Brillhart data point therefore reflects a rough attempt to show the true capability of this factorlzation technique.

Unquestionably, the most extensive -- and up to date -- compilation of integer factorizations ever made is the Cunningham Project Table [11] pub11shed by the AMS in 1983. As the authors say in the introduction, "The present tables are now at the limit of what can be done by factoring through 50 digits ..." The mid-1981 data point representing this benchmark in Figure 1 indicates the spread above and below this 50-digit figure accounted for by the variation in difficulty of specific numbers. Roughly speaking, the Cunningham Project Table established a well defined standard of the computational feasibility of factoring any 50-digit number in at most one day's computing time. This was the state of the art in the fall of 1982 when the quadratic sleve in the form originally proposed by Carl Pomerance [13] was Implemented by Davis and Holdridge at Sandia on a Cray 1S. The Sandia effort was prompted by the recognition by Simmons, Tony Warnock of Cray Research and Marvin Wunderlich that the Cray's ability to efficlently pipeline vector operations on vectors containing thousands of elements could be matched to the sieving operation that was the heart of the quadratic sleve factoring algorithm. The immediate results were start- 
11ng. A pair of 51 and 52-digit numbers - taken from the composite cofactor Ifst in the Cunningham Project Table that gave them a recognized certificate of difficulty - were factored in under two hours. This represented a speed improvement of better than an order of magnitude on the first attempt over what had been posstble only a year earlier when the Cunningham Project Table was sent to press. This algorithm, using a very memory efficlent Gaussian elfmination routine for blnary matrices devised by Parkinson and Wunderlich [16], was found to have a feasible range of 50-58 digits, i.e., it could factor up to 58-digit numbers in approximately a day's CPU time.

The next big advance occurred in 1983 when Davis discovered the speclal q variation to the basic quadratic sleve [10]. This innovation is so vital to the Sandia advances, and to the most recent factoring results shown In Figure 1, that it will be discussed in detail later. We also give some preclse cross-comparisons of the time required to factor numbers uslag the quadratic sleve, both with and whout the special q variation, later in this section, but roughly speaking this improvement bought another order of magnitude improvement in the speed of factoring.

The last two data points in Figure 1 are another factor of six or seven removed from the points in the error bar for the special q algorithm. This Is due to the optimization of the coding of the special q algorithm for the Cray -- attributable in large part to an Improved search algorithm developed by Tony Warnock. In addition, Holdridge found that by "unrolifng" the nested loops in the code the running times could be substantially improved. In other words, the six-fold improvement between the speclal q case and the 69 and 71-diglt examples 1 s primar1ly due to the substantially 1mproved efficlency with which the computers were coded and used. 
The research on factoring at the Sandia National Laboratorles has been proof tested at each stage of algorithm development on numbers that were left unfactored in [11] and which were clted as being of elther extraordinary Interest or difflculty to factor or both. For example, in [11], there is a table of the "Ten 'Most Wanted' Factorizations" that included as the first two entries the composite cofactors of the only two surviving unfactored composite numbers from Mersenne's 1640 11st; $2^{211-1}$ and $2^{251-1}$. Since this list was essentially an open challenge to the factoring community, we have responded by factoring all ten of them (nine of them for the first time). In the "shorthand" notation of [11], the numbers and the vital statistics of their factorization are shown in Table I. 2,211- C60 denotes the 60-digit composite cofactor of $2^{211}-1$, etc. The cofactors themselves are

Table I.

10 Most Wanted Factorizations

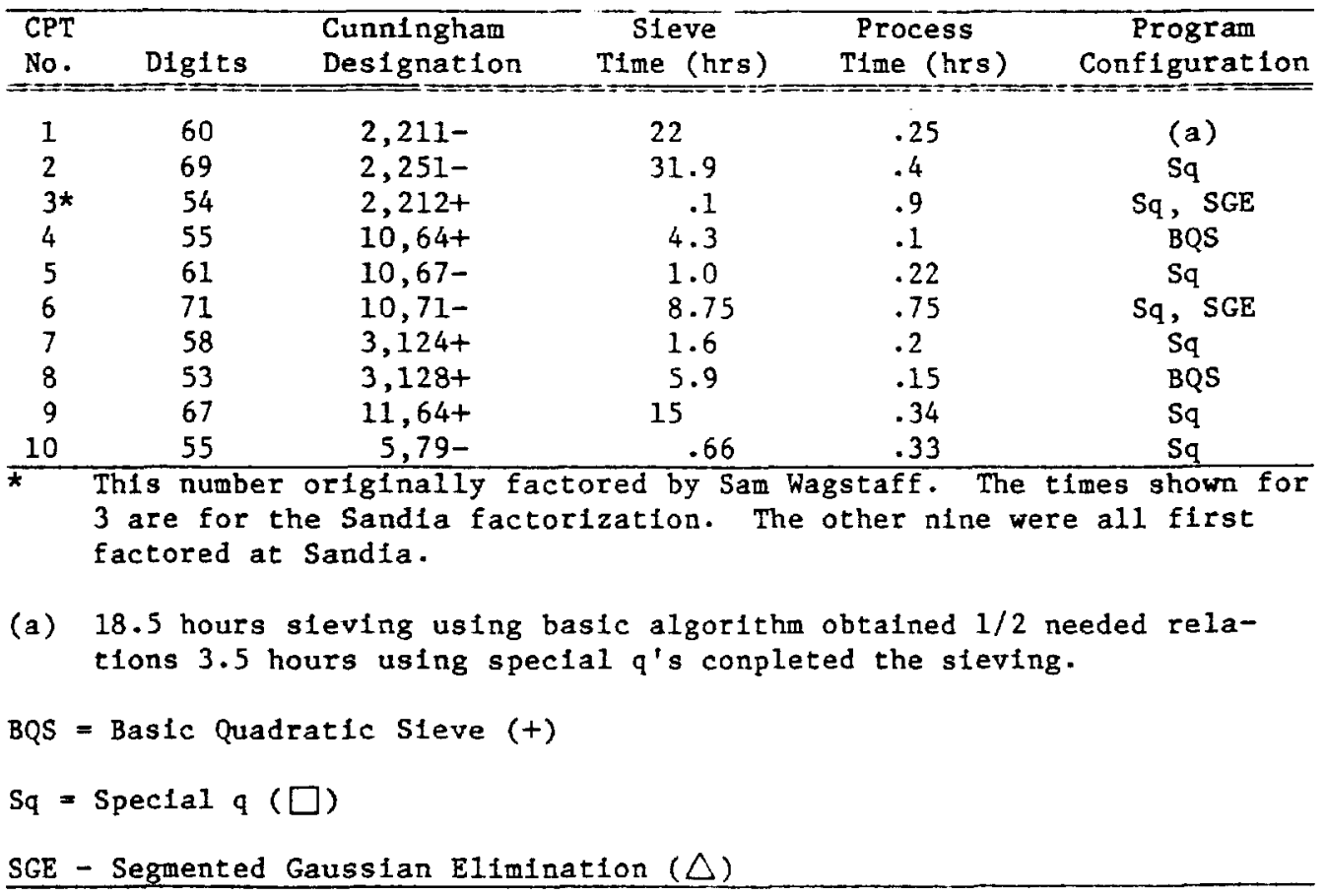


tabulated in [11]. Figure 2 plots the total computing time required to factor these numbers. As already mentioned, there have been three distinct generations of quadratic sieving algorithms, although refinements and improvements have occurred steadily in each generation of software. The + symbol denotes factorizations made with the original quadratic sieve, $\square$ the spectal $q$ algorithm and $\triangle$, the segmented (partioned matrix) Gaussian elimination codes that make it possible to handle much larger prime bases than would otherwise be possible. The 54 -digft outlier $(2,212+)$ is the result of factoring a small number using the pattitioned matrix code, so that almost all of the time shown was overhead spent in moving blocks of the matrix into and out of memory. It is included for completeness, but

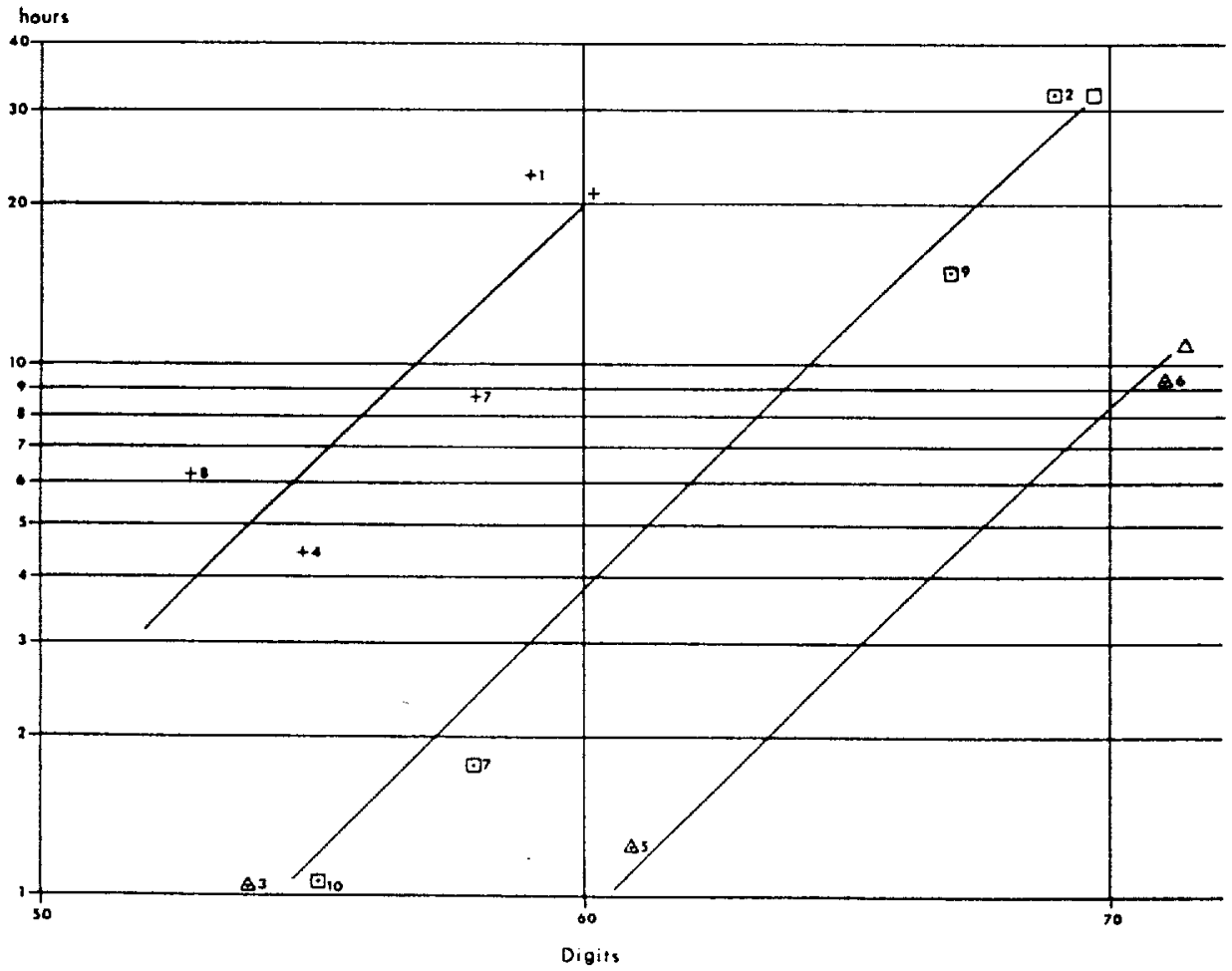


would have taken roughly the same time to factor using only the speclal $q$ algorithm. Incidentally, the approximating curves are simply fits of $(L(n))^{c}$ where $L(n)=e^{\sqrt{\log n \times \log \log n}}$ is the bound that most of the general purpose factoring algorithms seem to obey [13].

Especially noteworthy is the 58-diglt number (\#7, 3,124+ from [11]) that was Factored twice; first using the basic quadratic sieve in a time of 8.78 hours and then again using the spectal $q$ algorthm in 1.76 hours with a five-fold improvement in speed. This provides a crisp crosscomparison of the algorthms since both factorizations were done on the Cray is with codes developed by Holdridge within a very short time span. Hence, the improvement in this case is directly attributable to the mathematics (special $q$ algorithm). An even more spectacular, but also more difficult to interpret cross-comparison is possible. In [13] Pomerance discusses the benchmark 49-digtt number $\frac{3^{121}-1}{\left(3^{11}-1\right) 11617}$ factored by Sam Wagstaff in 70 hours of computing and projects that it might be possible by various refinements (such as the early abort technique) to reduce the running time to as little as 20 hours. The latest generation of Sandia algorithms factored this number in 4 minutes and 34 seconds: a ratio of 920 to 1 In computing time! Admlttedly, this timing comparison is hard to interpret since different machines and different factoring algorlthms were used, but the comparison supports our earlier statement that a roughly three-order of magnitude overall 1mprovement in the speed of factorization has been achleved. Other comparisons gield simllar results.

Twenty-five large numbers ( $>40$ digfts) have been factored at Sandla -plus many other smaller numbers for which the overhead obscures the time actually spent in factoring. Flgure 3 shows a least squares fit of $(L(n))^{c}$ to the data on numbers of at least forty diglts for the three generations of algorlthms; marked,$+ \square$ and $\triangle$ as before. Note, however, that in an 


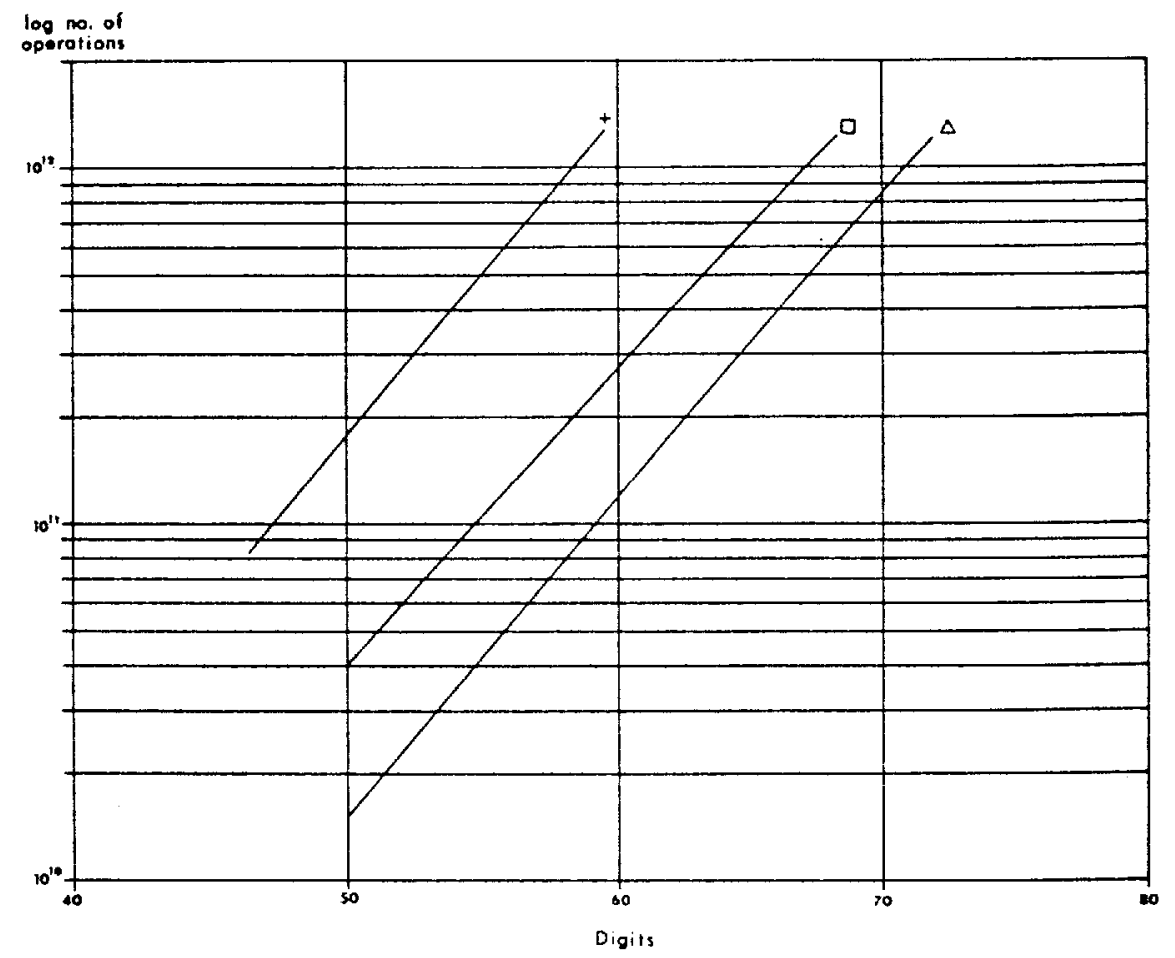

Figure 3.

effort to make the data machlne independent, we have plotted the number of elementary machine operations (shifts, adds, $X O R$, etc.) rather than the total time required to factor a number. The $\triangle$ curve is translated upward in Figure 3 compared to the same curve in Figure 2 since the Cray $X-M P$ has a basic clock frequency of $105 \mathrm{MHz}$ compared to the $80 \mathrm{MHz}$ clock frequency for the Cray 1S, so that the elapsed time (Figure 2) for a given number of elementary operations on the X-MP is roughly $3 / 4$ of what it would be on the 1S. The most obvious conclusion to be drawn from Figure 3 is that the Sandia work has - for a given number of machine operations -- roughly increased the size of the number that can be factored by thirteen digits. This may not sound like much of an improvement, but over the range from 40 to 75 digits -- essentially independent of the algorlthm used -- for each 
three-digit increase in the size of the number to be factored, the time required roughly doubles. This translates into slightly more than a 20fold Improvement in factoring resulting from the Sandia work, independent of the machine. This latter statement assumes that the machine is vectorized so that the quadratic sieve can be accommodated efficlently and also that the memory is organized in such a way that data can be "streamed" through an artthmetic unit and back into memory, etc., as is needed for an efficient implementation of a quadratic sieve. The Crays have this type of architecture, but so does the NEC SX-2, the Fujitsu VP-200 and the Hitach1 S-810 $[14,15]$.

A sort of "sound barrier" in computing is $10^{12}$ operations. At present this is a generally accepted dividing line between what is computationally feasible and infeasible. Figure 4, taken from the same data shown in Fig-

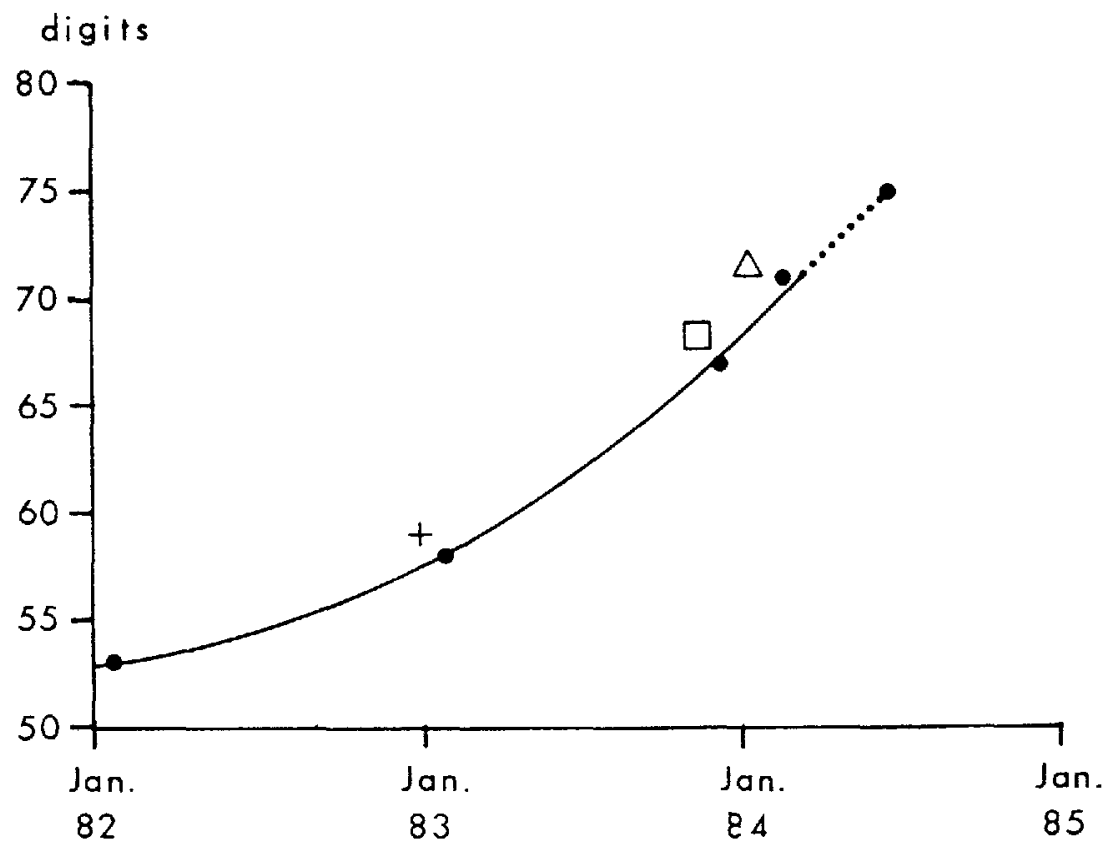

Figure 4. Size of composite "hard" number factorable by $10^{12}$ operations. 
ures 1 and 3 , shows how large a composite "hard" number could be factored using $10^{12}$ operations with the various generations of Sandia quadratic sleving algorfthms and codes. Again, the roughly thirteen-digit overall Improvement mentioned earlier can be seen to hold at $10^{12}$ operations decreasing to roughly ten digits improvement at $10^{10}$ operations -- the difference being due to the relative effect of the fixed overhead in the computation.

The third factor, in addition to the algorithmic improvements and the advances in the speed and power of the machines on which the algorithms are run, that has made a major contribution to speeding up the factorizat1on of large numbers is the architecture of the Cray family of computers (or of the Cray-1ike vectorized machines such as the NEC SX-2, the Fujitsu VP-200 and the Hitachf $S-810$ ). We presuppose here that the reader is either already acquainted with the essential steps in factoring using a quadratic sieve, or else that he will return to this portion of the paper after having read the detalled discussion of the algortthm steps. Roughly speaking there are three major time-consuming steps. One involves the subtraction of the logarithm of a prime number, $p_{i}$, from on the order of $\left(1 / P_{1}\right) \times 10^{10}$ locations for the largest numbers factored. Another requires forming the ring sum (exclusive $O R$, or $\Theta$ ) of a pafr of binary vectors 7-15,000 bits long several million times. The third task, which has often been described as searching for a needle in a $10^{9}$ haystack, is a search over $\approx 10^{10}$ locatlons looking for Iinear dependenctes, where we expect on average 20 "hits" in the $10^{10}$ items searched. To appreclate the 1mpact of the computer architecture on the speed of execution -- consider the first operation described above in which the same quantity, $-\log p_{i}$, is to be added to a string of memory locations that can be indexed in such a way that the locations to which $-\log P_{1}$ is to be added differ by a constant 
$p_{1}^{j}$. The total string length is $\approx 10^{10}$. In a machine of more or less conventional architecture in which data is fetched from memory, operated on in the arithmetic unit (AU) and the result then returned to memory, this sort of operation is slow. Programmed optimally on a CDC 7600 only a 1 megabit per second effective throughput is possible. The Cray however has the ablity to "stream" Information from memory through the $\mathrm{AU}$ and back into memory for a flxed operation without pausing for fetch, store or interpret states. As a result, we can carry out this operation,

$$
\begin{aligned}
& \text { DO } 10, I=J, N, P_{1}^{j} \quad \mathrm{~N}=10^{10} \\
& 10 \quad A(I)=A(I)-X\left(P_{1}\right),
\end{aligned}
$$

where $X\left(P_{1}\right)$ is the logarithm of $\mathrm{P}_{1}$, at $1 / 2$ the clock rate of $80 \mathrm{MHz}$ on the Cray is and at the full clock rate of $105 \mathrm{MHz}$ on the Cray $\mathrm{X}-\mathrm{MP}$. In other words, the architecture alone has accounted for a speed up of nearly forty times (Cray X-MP with $105 \mathrm{MHz}$ clock versus CDC 7600 with a $37 \mathrm{MHz}$ clock rate) for this type of operation! In order to exploit the capability to stream fnformation from the memory through the $\mathrm{AU}$ and back into memory, the algorithm must have many long strings on which a fixed operation needs to be performed. The recognition that quadratic sleving could be organlzed in such a way that this feature of the Crays could be explofted is what prompted the Sandia research in the first place.

The exclusive or operation

$$
A(I)=A(I) \oplus B(I)
$$

goes at the same rate as the subtraction, $1 . e$. , the Cray 1 s streams at $1 / 2 \times 80 \mathrm{MHz}$ while the Cray X-MP can stream data at $1 \times 105 \mathrm{MHz}$. The search operation in elther of the Crays has an overhead that only allows a throughput of $2 / 3$ of the clock rate, $1 . e ., 2 / 3 \times 80 \mathrm{MHz}$ for the Cray $1 \mathrm{~S}$ 
or $2 / 3 \times 105 \mathrm{MHz}$ for the Cray $\mathrm{X}-\mathrm{MP}$.

In addition to using the ability of the Crays to stream data, Holdridge did a timing analysis and found that if the major sleving loop was "unrolled" that the same computation could be carried out even faster.

As a result of the timing analysis of the sieving code it was also determined that a great deal of time was spent in searching. Once the sleving is done those vector entrles that have reached a specified $11 \mathrm{mit}$ must be found and saved. The search, written in Fortran with an "if" statement was not vectorized by the Cray compiler. The search is now done by a Cray Assembly Language (CAL) subroutine which does use the vectorization capability and Is much faster.

The bottom line, when all of these refinements are included and when one welghs the efficiencles for the various operations with the relative times spent in carrying out the associated calculation, is that the Cray 15, running the quadratic sieve, has a throughput (bits of meaningful information processed per second) of $1 / 4 \times 80 \mathrm{MHz}$ wh1le the Cray X-MP achieves $3 / 4 \times 105 \mathrm{MHz}$. Both of these figures are quite impressive since they indicate that the coding is exceptionally taut -- so much so that Cray scientists have sald that these codes come the closest to running the Crays "flat out" of any codes they know of. The point is that since no code can have a throughput greater than the clock rate, and since the throughput with these codes (espectally on the X-MP) is so close to the clock rate, there is only a marginal improvement possible from further refinements of the coding -- for the present factoring algorithms. Almost an order of magnitude of the total advance in factoring achieved at Sandia is attributable to the efficlency with which the Crays are belng used, 1.e., to the tautness of the codes.

We can extrapolate the future of factoring a short distance into the 
future with relatively high confidence. First, the Cray X-MP is a dual processor machine in which the present Sandia code has only used one of the processors. Preliminary work on splitting up the main parts of the quadratic sieving calculation so that two processors can be efficiently employed -- a nontrivial task incidentally -- suggests that 1 may be possible to gain a factor of $\approx 1.7$ in computing effectiveness by using the $X-M P$ to its fullest. Using the rule of thumb that doubling the computing time roughly equates to increasing the size of the number that can be factored with a fixed amount of work by three digits -- taking advantage of the dual processor capability of the Cray X-MP should make it possible to factor numbers of $73-74$ digtts in the same time required to factor the 71-digit number using a single processor. Another way of stating this result is; with the present code and using the Cray X-MP, 75-digit numbers should be factorable in roughly a day's computing time.

Looking at the next generation of vectorized machines -- especially the Cray II and also the Fujitsu VP-200 or the NEC SX-2 [14,15], a11 w111 have a 256 mfllion word high-speed memory compared to the four million word memory on the Cray $X$-MP used in the research reported here. The Cray II has a projected arithmetlc capability of 2000 megaflops (millions of floatIng point operations per second) while the Japanese machines have 533 and 1300 megaflops respectively compared to $\approx 100$ megaflops for the Cray $X-M P$. Perhaps more significantly for the quadratic sieve algorithm, all have an Improved vectorization capability; $80 \mathrm{~K}$ for the SX-2 and $64 \mathrm{~K}$ for the VP-200 compared to the $4 \mathrm{~K}$ capability of the Cray $1 \mathrm{~S}$ or $2 \times 4 \mathrm{~K}$ of the Cray $\mathrm{X}-\mathrm{MP}$. All of these factors when combined suggest that the Cray II and probably the Fujitsu VP-200 or the NEC $S X-2$ will be roughly elght to nine tines more effective in factoring using the quadratic sieve than is the Cray $X-M P$. This translates into an increase in the size of the numbers that can be factored of $\approx 10$ digits. We therefore feel quite confident in pro- 
jecting that 85-digit numbers will be factorable in a day's time using the machines that will be avallable in the next year or so as indicated in Figure 1.

Beyond that point, we leave it to the reader to draw his own conclusions. It is unlikely, however, that efther of the curves in Figures 1 or 4 showing recent progress in factoring will suddenly go "flat", but whether the exponential rate of change will continue is impossible to predict. What does appear plausible to predict, though, is that it will be feasible to factor 100 digits by the end of the decade, i.e., by 1990 .

\section{Fanciful Factoring}

Most general purpose factoring algorithms (continued fraction, Schroeppel's sleve and the various quadratic sieves) depend for their success on the following simple observation. In the ring of restdues modulo a composite number $n$, any quadratic residue, $y, i . e .$, a residue that is the square of some other element in the ring, has at least four "square roots" -- and perhaps many more depending on the cholce of $y$ and on the prime decomposition of $\mathrm{a}$. If there existed an oracle that when presented with a quadratic restdue, $y$, would pronounce a square root of $y$, then $n$ could be factored with probability that goes to 1 exponentially fast. For example, if $n=p q, p$ and $q$ distinct primes, and $y=x^{2}$ (mod $n$ ) where $x$ has the unique representation $x=a p+b q(\bmod n)$, where $0<a<q$ and $0<b<p$, then $y$ has the four square roots $( \pm a) p+( \pm b) q$ where we Interpret $-a=q-a$ and $-b=p-b$. To factor $n$ using the services of the oracle, choose $x=a p+b q$ (at random) and compute the quadratic residue $y$ $\equiv x^{2} \equiv a^{2} p^{2}+b^{2} q^{2}(\bmod n)$. We, of course, do not know $a, p, b$ or $q$ since we don't yet know the factorization of $n$, but we do know $x$ and $y$. The oracle when presented with $y$, would with probability $1 / 2$ pronounce efther $y^{1 / 2}=a p+b q$ or $y^{1 / 2}=(-a) p+(-b) q$ in whlch case we learn nothing 
about the factorization of $n$. On the other hand, with probability $1 / 2$ the oracle would pronounce elther $y^{1 / 2}=a p+(-b) q$ or $y^{1 / 2}=(-a) p+b q$. In which case the two greatest common divisors;

$$
\left(x+y^{1 / 2}, n\right) \text { and }\left(x-y^{1 / 2}, x\right)
$$

would be elther $p$ and $q$ or else $q$ and $p$, respectively, depending on which root the oracle chose.

All of the general purpose factoring algorithms mentloned cause the computer to function (ultimately) in the same way as our fancled oracle. The main difference is that instead of getting back a square root as the response to a submitted quadratic residue, the algorithm ylelds a sequence of Intermediate answers, that ultimately amount to one of the oracle's responses. Just as $1 n$ the case of the oracle, a quadratic residue, $Q_{1}$, is presented to the algorithm -- but the response is not (except in the rarest of cases) a square root of $Q_{1}$, but rather the prime decomposition of $Q_{1}$, In which some of the prime factors may occur to an odd power. Hence it is computationally infeasible to lnfer a square root of $Q_{1}$ from the response, since this would be equivalent to being able to factor $n$. If after sufficlent1y many responses, however, a subset of the $Q^{\prime}$ s can be found for which each of the primes that has occured as a factor in some one of the $Q^{\top} s$ has occurred an even number of times $f_{n}$ all, then we are able to effectively recreate one of the oracle's responses. Since the product of the Q's is a quadratic residue of a root that we know and the square root of the product of the primes is trivially the product of each of the primes raised to half of its even exponent, it is also feasible to calculate a square root. Just as in the case of the oracle, when $\mathbf{n}=\mathbf{p q}$ there is only a 50-50 chance that this will lead to a factorization of $n$ with comparable probabilities for other composite $n$, but this is the essential notion underlying the various factoring schemes. 
Quadratic Sieving: Plain

Given an odd number, $n$, to be factored, the basic quadratic sieving scheme [13] calculates a sequence of (relatively) small quadratic residues

$$
Q(x)=(x+m)^{2}-n
$$

where $m=[\sqrt{n}]$. If $|x|<B$ and $B \ll \sqrt{n}$, then $Q(x)$ will be "close" to $\sqrt{n}$. It is Important to keep $Q(x)$ small since the algorithm attempts to factor $Q(x)$ over a prescribed -- but restricted -- set of primes. This set of possible factors of $Q(x)$ consists of precisely those primes for which $n$ is a quadratic residue, $i . e,, 2$ and the odd primes, $p$, for which the Legendre symbol $(n / p)=1$. Fortunately, the Legendre symbol is easy to calculate in a manner similar to the Euclidean algorlthm for finding the greatest common divisior, so that it is computationally easy to find the $t-1$ smallest odd primes, $p_{1}$, for which $\left(n / p_{1}\right)=1$. The set of $t+1$ elements $\left(-1,2, p_{1}\right)$ we shall refer to as the factor base. In order for the algorithm to succeed, we must find sufficlently many quadratic residues, $Q(x)$, that factor completely into factors in the factor base so that it is possible to find some subset of the $Q(x)$ among which the prime factors have all occurred an even number of times. The justification for referring to the procedure as a sieve is now easy to see. If $p^{\alpha} \mid Q(x)$ for some $x$, then $\mathrm{p}^{\alpha} \mid\left(\mathrm{Q}\left(\mathrm{x} \pm \mathrm{hp} \mathrm{p}^{\alpha}\right), \mathrm{h}=0,1,2, \ldots\right.$, hence the division of the resulting sequence of quadratic residues can be performed by a sleve-like operation at argument values indexed in an arithmetic progression with spacing of $p^{\alpha}$. One of the primary reasons for the speed of the quadratic sieving algorithm is that instead of having to carry out multiple precision trial divisions as is required in some of the other general purpose factoring algorlthms, we can use single precision subtraction of approximate logarIthms on the $Q\left(x \pm \mathrm{p}^{\alpha}\right), f . e$. , at only those positions where it is 
already known that $p^{\alpha}$ is a divisor.

Since we must ultimately be able to combine a subset of the factored residues by multiplication to form a perfect square, 1.e., to simulate a response by the oracle, we need to find a linear combiation of the exponents for the primes appearing in the various factorizations such that the sum is even in each entry (for each prime). In order to have a reasonable chance of finding such a dependency we should have approximately as many completely factored residues as we have primes in the factor base. One might conclude from this, that $t$ should be small. However, if we take $t$ to be too small, then a given residue is not likely to factor. On the other hand, if we take $t$ to be too large, we spend more time sieving and w11 have to find many more factorizations in order to be able to find a linearly dependent subset. It is clear, though, that qualitatively speaking as the magnitude of $n$ increases, the number of entrles, $t$, in the factor base should also lncrease. If one had no storage constraints, it would be possible to optimize the size of the factor base in order to minimlze running time. In fact Wunderlich has analyzed, partly theoretically and partly empirically, the optimum size of the factor base as a function of the size of $n$, but the conclusion is that this optimum is so large that using a $t$ of this size would result in an impractically large matrix even for the Cray X-MP; hence, we simply use as large a factor base as we can accommodate.

A detalled discussion of the coded implementation of quadratic sieves Is inappropriate to the objectives of this paper, however, it is necessary to understand the essential steps involved in using sieves for factoring in order to apprectate why and how the Cray machines can be so well matched to the algorithm. For the $Q(x)$ defined in (1), we wish to find solutions, $x$, to the related congruences 


$$
Q(x) \equiv 0 \quad\left(\bmod P_{i}\right)
$$

for $P_{i}$ an element in the factor base. As already remarked (2) has solutions precisely when $\left(n / p_{i}\right)=1, p_{i} \mid n$ or $p_{1}=2$. If $\left(n / p_{i}\right)=1$ then $(2)$ has two solutions, which are usually represented as $A_{11}$ and $B_{11}=-\left(A_{11}+2 m\right)\left(\bmod P_{1}\right)$. The sleving process depends on the fact that if we had a list of consecutive values of $Q(x)$ indexed by $x$, that for all indices $x=A_{11} \pm h_{i}$ and $x=B_{11} \pm h p_{1}$ the associated $Q(x)$ would be divisabie by $P_{1}$. The sieving procedure consists of dividing out (effectively) $p_{i}$ from only these $Q(x)$ while leaving all other $Q(x)$ unaffected. Th1s requires two slevings of the array per solution to (2) -- but as we shall see, the whole procedure can be implemented very efficiently.

As a matter of fact, we actually wish to solve a slightly more general version of (2)

$$
Q(x) \equiv 0 \quad\left(\bmod p_{1}^{j}\right)
$$

since the smaller primes may occur to some power $>1$ in the factorization of $Q(x)$ over the factor base. We therefore choose a bound $L$ and steve for al1 $p_{1}^{j}<L$ where $p_{1}$ is in the factor base. We have generally taken $L$ to be the length of one sleving block $\left(8 \times 10^{5}\right.$ on the cray 1S). This gives at least one successful division per prime power per sleving interval.

For each odd prime $p_{f}$ in the factor base and each exponent $f$ such that $p_{1}^{j}<L$ compute and save the two integers $A_{1 j}$ and $B_{i j}$, that are obvious generalizations of the $A_{11}$ and $B_{11}$ defined in connection with (2). $A_{1 j}$ is the least nonnegative residue (mod $p_{1}^{j}$ ) that satisfies (3) and $B_{1 j}=-\left(A_{1 j}+2 m\right)\left(\bmod p_{j}^{j}\right)$ is its palred solution. These starting addresses for sleving are stored along with the associated weight $\log \mathrm{P}_{1}$. In the same way that $Q(x)$ was steved for $P_{1}$, we sleve at $x=A_{1 j} \pm h_{1} j$ and $x=B_{1 j} \pm h p_{1}^{j}$ by subtracting the weight $\log p_{1}, 1>1$. If $l$ is 
the highest power of $p_{1}$ that divides $Q(x)$ for some particular argument $x$ and $p_{1}^{\ell} \leqslant L$, then $\log p_{i}$ will be subtracted from $Q(x)$ precisely $\ell$ times as 1 th should be by this procedure.

The prime 2, of course, has every odd integer as a quadratic residue but $\mathrm{x}^{2} \equiv \mathrm{n} \bmod 4$ has a solution if and only if $\mathrm{n} \equiv 1 \bmod 4$. Also for $k>3, x^{2} \equiv n \bmod 2^{k}$ has solutions $1 f$ and only if $n \equiv 1 \bmod 8$. Thus, the indices for sleving with powers of 2 must be chosen in a somewhat different fashion depending on the residue class of $n \bmod 8$. Following a suggestion of Pomerance, these sleving parameters are assigned as follows:

In a11 cases

$$
\begin{array}{ll}
A_{11} \equiv(1-m) & (\bmod 2) \\
A_{12}, B_{11}, B_{12} & \text { undefined }
\end{array}
$$

The other values of $A_{1 j}, B_{1 j}$ must be treated as three distinct cases:

1) $n \equiv 1 \bmod 8$

For $j=3,4, \ldots, l, \frac{B}{2}<2^{\ell}<B, A_{1 j}$ is chosen such that

$$
\begin{aligned}
\left(A_{1 \cdot j}-m\right)^{2} & \equiv n \quad\left(\bmod 2^{j+1}\right) \quad \text { and } 0<A_{1 j}<2^{j} \\
B_{1 j} & \equiv-\left(A_{1 j}+2 m\right) \quad\left(\bmod 2^{j}\right) .
\end{aligned}
$$

$A_{11}$ is assigned weight $3 \log 2$. All other defined $A_{1 j}, B_{1 j}$ have weight $\log 2$.

2) $\mathrm{n} \equiv 3 \bmod 4$

$A_{11}$ is assigned weight $\log 2$. Other $A_{1 j}, B_{1 j}$ are undefined.

3) $\mathrm{n}=5 \bmod 8$

$A_{11}$ is assigned weight $2 \log 2$. Dther $A_{1 j}, B_{1 j}$ are undefined. 
In sieving, start from the origin $(x=0)$ and sieve in both positive and negative directions unt1l approximately $t$ of the $Q(x)$ have factored completely over the factor base. Because of some overhead in the 1nitiallzing of arrays and the pipelining capabilfty of the Cray's, we sieve on intervals which are as large as possible, say of length $k,[0, k),[-k, 0)$, $[k, 2 k), \ldots: k=765,000$ on the Cray 15 and $1.5 \times 10^{6}$ on the Cray $X-M P$.

In order to be able to carry out the factorization by subtraction, we need to first fill the arrays with approximate (single precision) values of $\log |Q(x)|, x \in[\ell k,(t+1) k)$. After the first positive and negative blocks, these logarithms are taken as constant in a given sieving interval. When an array has been Inftialized in this way, we sleve on $p_{1}^{j}$ by subtracting the assigned welght (usually $\log \mathrm{p}_{1}$ as discussed above) from each $\log Q(x)$ in the arithmetic progression of indices

$$
x=A_{1 j} \pm h p_{1}^{j} \quad \text { and } \quad x=B_{1 j} \pm h p_{1}^{j}
$$

and in the sleving interval $[k \ell, k(\ell+1))$.

When the sleving procedure is completed for a given block, the contents remaining in each location are compared with $\log p_{t}$ : where $p_{t}$ is the largest prime in the factor base. Values that are smaller then $\log \mathrm{Pt}$ Indicate the residues corresponding to these locations have been factored entirely into the primes in the factor base and these addresses are stored. Occasional false alarms due to approximations are eliminated later. Notice that very little multiple precision arithmetic is needed. The sleving procedure 1 s repeated block-by-block unt11 the desired number of Q's have been found that factored completely over the factor base. Once this has been done, it should be possible to find a subset of the Q's among which each of the primes has occurred an even number of times in total. The 
problem of finding such a subset can best be treated as a problem of findIng a linear dependency among vectors over GF(2). For each address $x_{1}$ at which $Q\left(x_{1}\right)$ factored completely over the factor base as indicated by the entry being less than $\log p_{t}, Q\left(x_{1}\right)$ is now actualig factored by dividing out the factors in the prime base to get

$$
Q\left(x_{i}\right)=\prod_{j=0}^{t} p_{j}^{\alpha_{1 j}}
$$

with which we assocfate a binary, $t+1$ element, vector $v_{1}=\left(v_{1 j}\right)$ where $v_{1 j}=1$ if $a_{1 j}$ is odd and 0 otherwise. This results in a roughly $t \times t$ binary array, in which all of the essential information concerning the factorization of each $Q\left(x_{1}\right)$ is stored in $t+1$ bits, or $2^{-6}(t+1)$ words in the Cray. Now we need to find a subset, $S$, of the $v_{1}$ such that $\underset{\mathrm{S}}{\oplus} \mathrm{v}_{1}=\phi$ where $\oplus$ denotes excluslve $\mathrm{OR}$ and $\phi$ denotes the zero vector. This is a stralghtforward problem in Gaussian elimination over GF(2). We use an improved version of a code developed by Parkinson and Wunderlich for this part of the calculation [16]. Once such a subset $\mathrm{S}$ is found, and $w$ and $z$ are calculated from

where

$$
w \equiv \prod_{S}(x+m) \quad(\bmod n)
$$

$$
Q(x)=(x+m)^{2}-n \text { and } Q(x) \& S \text {. }
$$

and

$$
z \equiv \prod_{j=0}^{t} \prod_{s} p_{j}^{\frac{1}{2} \Sigma \alpha_{1} j} \quad(\bmod n)
$$

if $w \pm z$, $n$ can be factored by calculating the greatest common divisors

$$
(w+z, n) \text { and }(w-z, n) \text {, }
$$


both of which will be proper divisors of $n$. It may be the case that nelther is a prime and that the procedure will have to be fterated to eventually obtain the prime decomposition of $n$, however the factor base remains the same and the factorizations of the $Q(x)$ have already been done, so that only the end calculation would need to be repeated with some other linear dependent subset.

\section{For Example}

Since this has been -- unavoldably -- a rather lengthy discussion, we illustrate the basic quadratic sieve factoring algorithm using a small example.

$$
\text { Let } \mathrm{a}=37 \cdot 137=5069 \text {, so that } \mathrm{m}=[\sqrt{5069}]=71 \text {. }
$$

This example was "cooked" so as to have many small prlmes in the factor base, 1.e., 5069 is a quadratic residue of $5,7,11$ and 13 , hence the factor base for $t=5$ consists of $-1,2,5,7,11,13$.

First $5069 \equiv 5(\bmod 8)$, so that $2^{2} \mid Q(0 \pm 2 h)$ and no other values of $Q(x)$ are divisable by a power of 2 from the earlier discussion of $A_{1 j}$ and $B_{1 j}$. Similarly, it is easy to show that for $P_{2}=5, A_{21}=1$ and hence that $B_{21}=-(1+2 \mathrm{~m}) \equiv-3 \equiv 2(\bmod 5)$. Thus $5 / Q(1 \pm 5 h)$ and $5 / Q(2 \pm 5 h)$, etc. Similar results hold for $p=7,11$ and 13 . Table II shows the

Table II

\begin{tabular}{|c|c|c|c|c|c|c|c|c|c|}
\hline $\begin{array}{c}x \\
x+a \\
Q(x)\end{array}$ & $\begin{array}{r}12 \\
72 \\
115\end{array}$ & $\begin{array}{r}2 \\
73 \\
260\end{array}$ & $\begin{array}{r}3 \\
74 \\
407\end{array}$ & $\begin{array}{r}4 \\
75 \\
556\end{array}$ & $\begin{array}{r}5 \\
76 \\
707\end{array}$ & $\begin{array}{r}6 \\
77 \\
880\end{array}$ & $\begin{array}{r}7 \\
78 \\
1015\end{array}$ & $\begin{array}{r}8 \\
79 \\
1172\end{array}$ & $\begin{array}{r}9 \\
80 \\
1331\end{array}$ \\
\hline $\begin{array}{l}\text { Pactors fron base } \\
\text { Reeidual }\end{array}$ & $\begin{array}{r}5 \\
23\end{array}$ & $22 \cdot 5 \cdot 13$ & $\begin{array}{l}11 \\
37\end{array}$ & $\begin{array}{r}2^{2} \\
139\end{array}$ & $\begin{array}{r}7 \\
101\end{array}$ & $\begin{array}{r}2 \cdot 5 \\
43\end{array}$ & $\begin{array}{r}5 \cdot 7 \\
29\end{array}$ & $\begin{array}{r}2^{2} \\
293\end{array}$ & $11^{3}$ \\
\hline$x$ & 0 & -1 & -2 & -3 & -4 & -5 & -6 & -7 & -8 \\
\hline $\begin{array}{r}x+m \\
Q(x)\end{array}$ & $\begin{array}{r}71 \\
-28\end{array}$ & $\begin{array}{r}70 \\
-169\end{array}$ & $\begin{array}{r}69 \\
-308\end{array}$ & $\begin{array}{r}68 \\
-445\end{array}$ & $\begin{array}{r}67 \\
-580\end{array}$ & $\begin{array}{r}66 \\
-713\end{array}$ & $\begin{array}{r}65 \\
-844\end{array}$ & $\begin{array}{r}64 \\
-973\end{array}$ & $\begin{array}{r}63 \\
-1100\end{array}$ \\
\hline $\begin{array}{l}\text { Factars frow base } \\
\text { Residual }\end{array}$ & $-1 \cdot 2^{2 \cdot 7}$ & $-1 \cdot 13^{2}$ & $-1 \cdot 2^{2} \cdot 7 \cdot 11$ & $\begin{array}{r}-1 \cdot 5 \\
89\end{array}$ & $\begin{array}{c}-1 \cdot 2^{2} \cdot 5 \\
29\end{array}$ & $\begin{array}{c}-1 \\
713 \\
(23 \cdot 31)\end{array}$ & $\begin{array}{r}-1 \cdot 2^{2} \\
211\end{array}$ & $\begin{array}{r}-1 \cdot 7 \\
139\end{array}$ & $-1 \cdot 2^{2} \cdot 5^{2} \cdot 11$ \\
\hline
\end{tabular}


shows the quadratic residues $Q(x)$ for $-8<x<9$, six of which factor completely over the factor base. The perlodic appearance of the factors on which sleving is based is easy to see. The corresponding binary matrix $V$ is of the form.

\begin{tabular}{l|rrrrrr}
\multicolumn{1}{c|}{} & -1 & 2 & 5 & 7 & 11 & 13 \\
\cline { 2 - 6 }$v_{9}$ & & & & 1 & \\
$v_{2}$ & & & 1 & & & 1 \\
$v_{0}$ & 1 & & 1 & & \\
$v_{-1}$ & 1 & & & & \\
$v_{-2}$ & 1 & & 1 & 1 & \\
$v_{-8}$ & 1 & & & 1 &
\end{tabular}

Three subsets of the $\nabla_{i}$ sum (exclusive OR) to $\phi:$

$$
\begin{aligned}
& \mathrm{v}_{0} \oplus \mathrm{v}_{-1} \oplus \mathrm{v}_{-2} \oplus \mathrm{v}_{-8}=\phi \\
& \mathrm{v}_{9} \oplus \mathrm{v}_{-1} \oplus \mathrm{v}_{-8}=\phi \\
& \mathrm{v}_{9} \oplus \mathrm{v}_{0} \oplus \mathrm{v}_{-2}=\phi
\end{aligned}
$$

The relationship in (a) corresponds to having presented the quadratic residue 3625 to the oracle:

$$
\begin{aligned}
3625 & \equiv Q(0) Q(-1) Q(-2) Q(-8) \quad(\bmod 5069) \\
& \equiv(71)^{2}(70)^{2}(69)^{2}(63)^{2} \quad(\bmod 5069) .
\end{aligned}
$$

In this case the algorithm (oracle) returns the result that

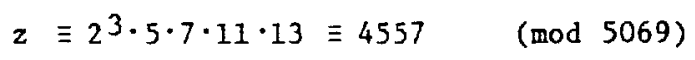

while we can calculate

$$
w \equiv 71 \cdot 70 \cdot 69 \cdot 63 \equiv 512 \quad(\bmod 5069)
$$

which tells us nothing whatsoever about the factorlzation of $n$, since $4557 \equiv-512(\bmod 5069)$. 
If however we use elther of the other two linear dependencles, we have: corresponding to b;

$$
\begin{aligned}
& 80 \cdot 70 \cdot 63 \neq \pm 2 \cdot 5 \cdot 11^{2} \cdot 13 \quad(\bmod 5069) \\
& 3039 \neq \pm 523 \quad(\bmod 5069)
\end{aligned}
$$

and hence

$$
\begin{aligned}
& (3039+523,5069)=137 \\
& (3039-523,5069)=37 .
\end{aligned}
$$

While corresponding to $c$;

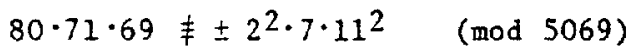

$$
\begin{aligned}
& 1067 \neq 3388 \quad(\bmod 5069)
\end{aligned}
$$

and hence

$$
\begin{aligned}
& (3388+1607,5069)=37 \\
& (3388-1607,5069)=137
\end{aligned}
$$

efther of which leads to the factorization of $n$.

It is this "plain" quadratic sleve factoring algorithm that was first implemented at Sandia, with the results documented in earlier portions of this paper. In attempting to use this technique to factor numbers larger than 57 to 58 digits, it was found that as the sieving interval became large enough to flad $\approx t$ residues that factored completely that the magnitudes of the quadratic residues to be factored themselves became prohibitively large. Eventually the frequency with which a quadratic residue could be completely factored over the prime base became so small that the sleving times were intolerable. For the largest numbers factored, we were examining many tens of millions of residues to find even one complete factorization. 


\section{Quadratic Sieving: Fancy}

If 1 it is the case that after all of the prime factors from the prime base have been factored out of a quadratic residue,

$$
Q(x)=q \prod_{1=0}^{t} p_{1}^{\alpha_{1}}
$$

the residual factor, $q$, is bounded by $p_{t}<q<p_{t}^{2}$, then $q$ is necessarily a prime. Use of these "large" primes in the factorization by simply adding them to the prime base has been suggested and implemented [13], since if two quadratic residues, $Q\left(x_{1}\right)$ and $Q\left(x_{2}\right)$ can be found such that

$$
Q\left(x_{1}\right) \equiv q \prod_{t=0}^{t} p_{i}^{\alpha_{11}} \quad(\bmod n),
$$

and

$$
Q\left(x_{2}\right) \equiv q \prod_{1=0}^{t} p_{1}^{\alpha_{21}} \quad(\bmod n)
$$

then

$$
Q\left(x_{1}\right) \cdot\left(x_{2}\right) q^{-2} \equiv \prod_{i=0}^{t} p_{i}^{\alpha_{1 i}+\alpha_{2 i}} \quad(\bmod n)
$$

1.e., a quadratic residue that can be factored over the prime base can be constructed.

Although this approach, known as the large prime varlation, does Improve the performance of the algortthm over the "plain" quadratic sieve, the 1mprovement isn't great enough to asymptotically make any difference. The reason that one only gets a marginal improvement from augmenting the prime base wh a large prime is that for all intents and purposes we are randomly generating the quadratic residues -- at least so for as their divisability by a particular prime is concerned. Therefore the probability 
that we will find another quadratic residue, $Q\left(x^{\prime}\right),-$ by sequentially indexing on $x$-- such that

$$
q \mid Q\left(x^{\prime}\right)
$$

is $\approx 1 / q$ per trial, which is a very small quantity. If Instead of simply searching for a $Q\left(x^{\prime}\right)$ such that $q \mid Q\left(x^{\prime}\right)$, we could systematically generate a new sequence of $Q^{\prime} s$, such that $q \mid Q(x)$, and in which $Q(x) / q$ is small, then we could recover the same comparatively high probability that the resulting quotients would completely factor over the factor base that we had for $Q(x)$ when $x$, and the $Q(x)$, were small. This is the essential idea behind Davis' special q variation or "quadratic sieving; fancy."

Assume that we have found in the regular quadratic sleving an $x$ for which

$$
Q(x)=q \prod_{1=0}^{t} p_{1}^{\alpha_{1}}
$$

where $\mathrm{p}_{\mathrm{t}}<\mathrm{q}<\mathrm{p}_{\mathrm{t}}^{2}$

A candidate for such an $x$ is found when the quantity remaining in one of the indexed entries after the sieveing is completed 1 les between $\log \mathrm{p}_{\mathrm{t}}$ and $2 \log p_{t}$. If by chance some of these candidate $Q(x)$ 's actually factor over the factor base because of large prime powers that were not considered In the original sleving, they are identffied later and Included among the complete factorizations. If $\mathrm{q}$ is actually prime, which is almost always the case, then note that:

$$
Q(x \pm h q)=(x \pm h q+m)^{2}-n=Q(x) \pm 2 h q(x+m)+h^{2} q^{2} \text {. }
$$

where each term on the right is divisible by $q$, and the magnitude $Q(x+h q) / q$ is essentially that of $Q(h), i . e ., \frac{Q(x \pm h q)}{q} \approx 2 h m$ for $x \ll \sqrt{n}$. We can then form subsequences of residues starting at $x$ and at $-(x+2 m)$ (mod $q$ ) 
whose magnttudes are comparable to those of $Q(x)$ at the start of the original sieve. The sieving on the subsequences is done exactly as in the plain quadratic sieve. One problem that may be encountered when using special q's is that the arguments may become larger than single precision words in the computer. We overcame this by using the pairs $(x, h)$ to represent $x+h q$ and thus temporarily avold multiprecision operations.

These special q's are relatively easy to find compared to finding complete factorizations. Thus in order to keep reduced residues "small", for each special $q$ we sieve the subsequence for only a short interval: typically for a few blocks. When dealing with a single special q any complete factorization of a quadratic residue contains the factor $q$, which can be eliminated before going to the Gaussian reduction by combining pairs of factorizations to get quadratic residues in which $q$ occurs an even number of times.

The sleving property is not dependent on the primality of the divisor, $q$, so why require the special q's to be prime? This is to prevent "collisions" between special q subsequences; that is, to prevent the same factorlation being generated by two subsequences. If $q_{1} \mid Q(x)$ and $q_{2} \mid Q(x)$ and $p_{t}^{2}>q_{1}>q_{2}>p_{t}$, then we would have $q_{1} \cdot q_{2} \mid Q(x)$. But then $Q(x)$ could not have passed the factorization criterion in the first place.

The special q modification introduced a few complications to the computation such as multiprecision arguments, and required writing a new computer code, but the increased capability was dramatic. The bottom line is that the special q vartation enabled factorization of 63-64 digit integers in times comparable to those required by the original sieve to Factor 55-56 digits. Furthermore, the relatively constant success rate for complete factorizations within the subsequences enables an accurate 
early estimation to be made of how much computing time will be required for a given factorization.

Examining the residuals in Table II we see that 23,29 and 31 are all candidate spectal $q^{\prime} s$. We let $q=23$ be the spectal $q$ in the example used earlfer. Table III shows the resulting quadratic residues $S_{x}$ based

Tab1e III

$$
\begin{gathered}
\text { Sieve on spectal } q=23 \\
Q^{\prime}(x)=Q\left(x_{0}+x q\right)=\left(x_{0}+x q+m\right)^{2}-n
\end{gathered}
$$

\begin{tabular}{|c|c|c|c|c|c|c|c|c|c|c|}
\hline \multirow[b]{2}{*}{$\begin{array}{c}x \\
x_{0}+x q+m \\
Q^{\prime}(x) / q\end{array}$} & \multicolumn{5}{|c|}{$\begin{array}{c}s_{x} \\
x_{0}=1\end{array}$} & \multicolumn{5}{|c|}{$\begin{array}{l}T_{X} \\
2 m) \equiv-5(\bmod 23)\end{array}$} \\
\hline & $\begin{array}{r}-2 \\
26 \\
-191\end{array}$ & $\begin{array}{r}-1 \\
49 \\
-116\end{array}$ & $\begin{array}{r}0 \\
72 \\
5\end{array}$ & $\begin{array}{r}1 \\
95 \\
172\end{array}$ & $\begin{array}{r}2 \\
118 \\
385\end{array}$ & $\begin{array}{r}-2 \\
20 \\
-203\end{array}$ & $\begin{array}{r}-1 \\
43 \\
-140\end{array}$ & $\begin{array}{r}0 \\
66 \\
-31\end{array}$ & $\begin{array}{r}1 \\
89 \\
124\end{array}$ & $\begin{array}{r}2 \\
112 \\
325\end{array}$ \\
\hline $\begin{array}{l}\text { Factors from base } \\
\text { Residual }\end{array}$ & $\begin{array}{r}-1 \\
191\end{array}$ & $\begin{array}{c}-1 \cdot 2^{2} \\
29\end{array}$ & 5 & $2^{2^{2}}$ & $5 \cdot 7 \cdot 11$ & $\begin{array}{r}-1 \cdot 7 \\
29\end{array}$ & $-1 \cdot 2^{2 \cdot 5 \cdot 7}$ & $\begin{array}{l}-1 \\
31\end{array}$ & $\begin{array}{l}2^{2} \\
31\end{array}$ & $5^{2} \cdot 13$ \\
\hline
\end{tabular}

on $x_{0}=1$ and $T_{x}$ based on the paired $x_{0} \equiv-(1-2 m) \equiv-5(\bmod 23)$ for $|x|<2$. Four residues factor completely over the prime base - extended by 23 .

\begin{tabular}{l|rrrrrrr}
\multicolumn{1}{c}{} & -1 & 2 & 5 & 7 & 11 & 13 & 23 \\
\cline { 2 - 7 }$S_{2}$ & & & 1 & 1 & 1 & & 1 \\
$S_{0}$ & & & 1 & & & & 1 \\
$T_{-1}$ & 1 & & 1 & 1 & & & 1 \\
$T_{2}$ & & & & & & 1 & 1
\end{tabular}

Eliminating 23 by multiplying each row by the first we get; 


\begin{tabular}{l|llllll} 
& -1 & 2 & 5 & 7 & 11 & 13 \\
\cline { 2 - 6 } $\mathrm{s}_{2} \mathrm{~S}_{0}$ & & & & 1 & 1 & \\
$\mathrm{~s}_{2} \mathrm{~T}_{-1}$ & 1 & & & & 1 & \\
$\mathrm{~s}_{2} \mathrm{~T}_{2}$ & & & 1 & 1 & 1 & 1
\end{tabular}

Referring to Table II, we see that

$$
\begin{aligned}
\mathrm{v}_{0} \oplus \mathrm{v}_{-8} \oplus \mathrm{s}_{2} \mathrm{~s}_{0}=\phi \\
\mathrm{v}_{-1} \oplus \mathrm{v}_{-2} \oplus \mathrm{s}_{2} \mathrm{~s}_{0}=\phi \\
\mathrm{v}_{-1} \oplus \mathrm{v}_{0} \oplus \mathrm{v}_{9} \oplus \mathrm{s}_{2} \mathrm{~s}_{0}=\phi \quad .
\end{aligned}
$$

From (a) we find

$$
w=71 \cdot 63 \cdot 118 \cdot 72 \equiv 315 \quad(\bmod 5069)
$$

and

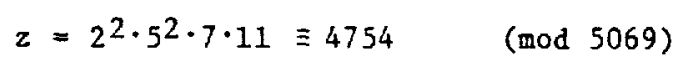

where

$$
w \equiv-z \quad(\bmod 5069) \quad
$$

Similarly from (b) we find

$$
w=70 \cdot 69 \cdot 118 \cdot 72 \equiv 2125 \quad(\bmod 5069)
$$

and

$$
z=2 \cdot 5 \cdot 7 \cdot 11 \cdot 13 \cdot 23 \equiv 2125 \quad(\bmod 5069)
$$

nelther of which tells us anything about the factorization of $n$. On the other hand, from (c)

$$
w=70 \cdot 71 \cdot 80 \cdot 118 \cdot 72 \equiv 2655 \quad(\bmod 5069)
$$

and

$$
z=2 \cdot 5 \cdot 7 \cdot 11^{2} \cdot 13 \cdot 23 \equiv 3099 \quad(\bmod 5069)
$$


where $w \pm z$. Hence

$$
(3099+2655,5069)=137
$$

and

$$
(3099-2655,5069)=37 .
$$

One cannot expect such a small example to fllustrate the advantages of using special q's -- although the range of the parameters is slight1y smaller in the example with the special $q$ than without.

\section{References}

1. R. Rivest, A. Shamir and L. Adleman, "A Method for Obtaining Digital Signatures and Public-key Cryptosystems," Commun. ACM 21, 2 (Feb. 1978), 120-126

2. H. Ong and C. P. Schnorr, "Signatures through Approxfmate Representations by Quadratic Forms," Proceedings of Crypto 83, Santa Barbara, CA, August 21-24, 1983, to be published by Plenum Press .

3. H. Ong, C. P. Schnorr and A. Shamir, "An Efflcient Signature Scheme Based on Quadratic Equations," to appear Proceedings of 16th Symposium on Theory of Computing, Washington D.C., Apri1 1984.

4. C. P. Schnorr, "A Cubic OSS-S1gnature Scheme," private communication, May 1984.

5. S. Even, O. Goldreich and A. Lempel, "A Randomlzed Protocol for Signing Contracts," In Advances in Cryptology, Proceedings of Crypto 82, Ed. by David Chaum, Ronald L. Rivest and Alan T. Sherman, Plenum Press, New York (1983).

6. G. J. Simmons, "The Prisoners' Problem and the Subliminal Channel," Proceedings of Crypto 83 , Santa Barbara, CA, August 21-24, 1983, to be published by Plenum Press. 
7. P. D. Merillat, "Secure Stand-Alone Posftive Personnel Identity Verification System (SSA-PPIV)," Sandia National Laboratories Tech. Rpt. SAND79-0070 (March 1979).

8. G. J. Simmons, "A System for Verifying User Identity and Authorfzation at the Polnt-of-Sale or Access," Cryptologia, Do1. 8, No. 1 (January, 1984), Pp . 1-21.

9. G. J. Simmons, "Verification of Treaty Compliance -- Revisited," Proceedings of the 1982 Symposium on Security and Privacy, Oakland, CA (Apr11 25-27, 1983), pp. 6I-66.

10. J. A. Davis and D. B. Holdridge, "Factorization Using the Quadratic Sieve Algorithm," Sandia National Laboratories Tech. Rpt. SAND83-1346 (Dec. 1983).

11. J. Brilihart, D. B. Lehmer, J. L. Selfridge, B. Tuckerman and S. S. Wagstaff, Jr., Factorizations of $b^{n} \pm 1 b=2,3,5,6,7,10,11,12$ up to High Powers," AMS Contemporary Mathematics, Vol. 22 (1983).

12. M. A. Morrison, J. Brillhart, "A Method of Factoring and the Factorization of $\mathrm{F}_{7}, "$ Math. Comp. 29 (1975), 183-205.

13. C. Pomerance, "Analysis and Comparison of Some Integer Factoring Algorithms," In Number Theory and Computers, Ed. by H. W. Lenstra, Jr., and R. Tijdeman, Math. Centrum Tracts, No. 154, Part I, Amsterdam (1982), pp. 89-139.

14. R. H. Mendez, "The Japanese Supercomputer Challenge," SIAM News, Vol. 17, No. 1 (January 1984), pP. 1 and 5 .

15. R. H. Mendez, "Benchmarks on Japanese and American Supercomputers -Preliminary Results," IEEE Trans. Comp, Vol. C-33, No. 4 (Apr11 1984). pp. $374-375$.

16. D. Parkinson, M. C. Wunderlich, "A Memory Efficient Algorithm for Gaussian Elimination over GF(2) on Parallel Computers," private communlcation (Feb. 1983). 